\title{
Semimetric Thresholds for Finite Posets
}

\author{
Yutaka Nakamura *
}

May 10, 2001

\begin{abstract}
Let $\succ$ be a binary relation on a finite set $X$. This paper proves that $\succ$ is irreflexive and transitive if and only if there is a real valued function $u$ on $X$ and a semimetric $\Omega$ on $X$ such that, for all $x, y \in X$, $x \succ y \Leftrightarrow u(x)>u(y)+\Omega(x, y)$.
\end{abstract}

\section{Introduction}

Let $\succ$ be an asymmetric binary relation on a set $X$ with symmetric complement $\sim$ : for all $x, y \in X, x \sim y$ if $\neg(x \succ y)$ and $\neg(y \succ x)$. When $\succ$ is acyclic (i.e., the transitive closure of $\succ$ is transitive), the simple relational system $(X, \succ)$ will be referred to as an acyclic set. When $\succ$ is irreflexive and transitive, $(X, \succ)$ will be referred to as a poset (partially ordered set).

It is proved by Bridges (1983) that if $X$ is countable, then $(X, \succ)$ is an acyclic set if and only if the following numerical representation holds: there is a real valued function $u$ on $X$ such that, for all $x, y \in X$,

$$
x \succ y \Rightarrow u(x)>u(y) .
$$

This "one-way" representation is undesirable because preferences are not recovered from the numerical representation $u$.

Several recent studies uncovered "two-way" representations for acyclic sets $(X, \succ)$, i.e., the numerical representations also reconstruct qualitative relation $\succ$. Abbas and Vincke (1993) and Agoev and Aleskerov (1993) considered finite acyclic sets and obtained the following two-way representation: there exist a real valued function $u$ and a real valued bivariate function $\Omega \geq 0$ on $X \times X$ such that, for all $x, y \in X$,

$$
x \succ y \Leftrightarrow u(x)>u(y)+\Omega(x, y) .
$$

\footnotetext{
*Institute of Policy and Planning Sciences, University of Tsukuba, 1-1-1 Tennoudai, Tsukuba, Ibaraki 305-8573, Japan; e-mail: nakamura@shako.sk.tsukuba.ac.jp; Tel. 029853-5547; Fax. 0298-53-5070
} 
Since $\Omega$ can be interpreted as a threshold, this representation will be dubbed here a bivariate threshold representation. Rodríguez-Palmero (1997) provided sufficient qualitative conditions for the representation when $X$ is a second countable topological space. A complete qualitative characterization of the representation for arbitrary $X$ was obtained by Diaye (1999). Nakamura (2000) developed several necessary and sufficient qualitative conditions for the existence of the representation when $X$ is the power set of a finite set. Other type of two-way representations for acyclic sets may be possible. For example, Subiza (1994) represents acyclicity by means of set-valued real functions.

The aim of the paper is to prove a similar bivariate threshold representation for finite posets. We show that $(X, \succ)$ is a poset if and only if $(X, \succ)$ has a bivariate threshold representation with $\Omega$ a semimetric on $X$, defined below, which is called a semimetric threshold representation. Posets may be more important than acyclic sets in many applications. However, there have been proposed and characterized no two-way representation of posets except Herrero and Subiza (1999), who represented arbitrary posets by means of set-valued real functions.

\section{The Main Theorem}

A semimetric ${ }^{1} \Omega$ on a set $X$ is a real valued function on $X \times X$ that satisfies the following three properties, understood as applying to all $x, y, z \in X$,

(1) $\Omega(x, x) \geq 0$

(2) $\Omega(x, y)=\Omega(y, x)$,

(3) $\Omega(x, y)+\Omega(y, z) \geq \Omega(x, z)$.

We note by (1) and (3) that $\Omega(x, y) \geq 0$ for all $x, y \in X$. The property (3) is called the triangle inequality.

Our main theorem is stated as follows.

Theorem 1 Suppose that $X$ is finite. Then $(X, \succ)$ is a poset if and only if there exist a real valued function $u$ on $X$ and a semimetric $\Omega$ on $X$ such that, for all $x, y \in X$,

$$
x \succ y \Leftrightarrow u(x)>u(y)+\Omega(x, y) .
$$

When a semimetric $\Omega$ is additively separable, i.e., for all $x, y \in X$,

$$
\Omega(x, y)=\omega(x)+\omega(y)
$$

for a nonnegative real valued function $\omega$ on $X$, the semimetric threshold representation characterizes special posets known as interval ordered sets.

\footnotetext{
${ }^{1} \mathrm{~A}$ metric is a semimetric that has the property that $\Omega(x, y)=0$ if and only if $x=y$.
} 
Nakamura (2001) provided a complete qualitative characterization of the representation for arbitrary $X$.

To prove the theorem, we use the following version of the familiar lemma for the existence of a solution to a finite system of linear inequalities (see Fishburn, 1970). Given two $N$ dimensional vectors of real numbers, $a=$ $\left(a_{1}, \ldots, a_{N}\right)$ and $b=\left(b_{1}, \ldots, b_{N}\right)$, we denote the inner product by $a \cdot b=$ $\sum_{i=1}^{N} a_{i} b_{i}$. A real vector is called rational if each component is a rational number, and is called integral if each of its components is an integer.

Lemma 1 Let $a^{1}, \ldots, a^{M}$ be $N$ dimensional rational vectors and $1 \leq K \leq$ $M$. Then either there is an $N$ dimensional integral vector $\rho$ such that

$$
\begin{aligned}
& \rho \cdot a^{k}>0 \quad \text { for } k=1, \ldots, K, \\
& \rho \cdot a^{k} \geq 0 \text { for } k=K+1, \ldots, M,
\end{aligned}
$$

or else there are nonnegative integers $\alpha_{1}, \ldots, \alpha_{M}$, with $\alpha_{k}>0$ for some $k \leq K$, such that

$$
\sum_{k=1}^{M} \alpha_{k} a_{j}^{k}=0 \text { for } j=1, \ldots, N .
$$

Note that the last equations in the lemma are described in the vector form by

$$
\sum_{k=1}^{M} \alpha_{k} a^{k}=0,
$$

where 0 is an $N$ dimensional zero vector. Since this equation says that some of $a^{1}, \ldots, a^{M}$ are linearly dependent, we shall call it the linearly dependent (LD) equation.

Proof of Theorem 1 If $(X, \succ)$ has a semimetric threshold representation, then it easily follows that $(X, \succ)$ is a poset. We shall assume henceforth that $X=\left\{x_{1}, \ldots, x_{n}\right\}$ is a nonempty finite set and that $(X, \succ)$ is a poset.

To specify our system of linear inequalities, suppose that $(X, \succ)$ has a semimetric threshold representation with a real valued function $u$ on $X$ and a semimetric $\Omega$ on $X$ satisfying

(1a) $u(x)-u(y)-\Omega(x, y)>0$ for all $x, y \in X$ such that $x \succ y$.

(1b) $u(x)-u(y)+\Omega(x, y) \geq 0$ and $u(y)-u(x)+\Omega(x, y) \geq 0$ for all $x, y \in \mathrm{X}$ such that $x \sim y$.

For real valued functions, $u$ on $X$ and $\Omega$ on $X \times X$, we define an $n$ dimensional row vector $\rho_{1}$ and a $\frac{1}{2} n(n+1)$ dimensional row vector $\rho_{2}$ by

$$
\begin{aligned}
\rho_{1}= & \left(u\left(x_{1}\right), \ldots, u\left(x_{n}\right)\right), \\
\rho_{2}= & \left(\Omega\left(x_{1}, x_{1}\right), \Omega\left(x_{2}, x_{1}\right), \Omega\left(x_{2}, x_{2}\right), \ldots,\right. \\
& \left.\quad \Omega\left(x_{n}, x_{1}\right), \Omega\left(x_{n}, x_{2}\right), \ldots, \Omega\left(x_{n}, x_{n}\right)\right) .
\end{aligned}
$$


For all $x, y \in X$, we define two column vectors, $\theta(x)$ with dimension $n$, and $\tau(x, y)$ with dimension $\frac{1}{2} n(n+1)$ as follows: for $k=1, \ldots, n, \ell=1, \ldots, n$, $i=1, \ldots, n$ and $j=1, \ldots, \frac{1}{2} n(n+1)$, the $i$-th component of $\theta\left(x_{k}\right)$ and the $j$-th component of $\tau\left(x_{k}, y_{\ell}\right)$ are given by

$$
\begin{aligned}
\theta_{i}\left(x_{k}\right) & = \begin{cases}1 & \text { if } i=k, \\
0 & \text { otherwise },\end{cases} \\
\tau_{j}\left(x_{k}, y_{\ell}\right) & = \begin{cases}1 & \text { if } j=\frac{1}{2} k(k-1)+\ell \text { and } k \geq \ell, \\
1 & \text { if } j=\frac{1}{2} \ell(\ell-1)+k \text { and } k<\ell, \\
0 & \text { otherwise. }\end{cases}
\end{aligned}
$$

We note that $\theta$ and $\tau$ are unit vectors, and $\tau(x, y)=\tau(y, x)$ for all $x, y \in X$.

Now we specify the system of linear inequalities for (1a) and (1b). Enumerate $\succ$ as $\left(x^{1}, y^{1}\right), \ldots,\left(x^{L_{1}}, y^{L_{1}}\right)$, half of $\sim$ as $\left(z^{1}, w^{1}\right), \ldots,\left(z^{L_{2}}, w^{L_{2}}\right)$ by using one of $(x, y)$ and $(y, x)$ when $x \sim y$, and $X \times X \times X$ as $\left(a^{1}, b^{1}, c^{1}\right), \ldots$, $\left(a^{L_{3}}, b^{L_{3}}, c^{L_{3}}\right)$. Then letting $\rho=\left(\rho_{1}, \rho_{2}\right)$ be a $\frac{1}{2} n(n+3)$ dimensional row vector, our system of linear inequalities are stated as follows:

(a)

(b)

$$
\rho \cdot\left[\begin{array}{c}
\theta\left(x^{i}\right)-\theta\left(y^{i}\right) \\
-\tau\left(x^{i}, y^{i}\right)
\end{array}\right]>0 \quad \text { for } i=1, \ldots, L_{1},
$$

(c)

$$
\begin{array}{ll}
\rho \cdot\left[\begin{array}{c}
\theta\left(z^{i}\right)-\theta\left(w^{i}\right) \\
\tau\left(z^{i}, w^{i}\right)
\end{array}\right] \geq 0 & \text { and } \\
\rho \cdot\left[\begin{array}{c}
\theta\left(w^{i}\right)-\theta\left(z^{i}\right) \\
\tau\left(z^{i}, w^{i}\right)
\end{array}\right] \geq 0 & \text { for } i=1, \ldots, L_{2},
\end{array}
$$

$$
\rho \cdot\left[\begin{array}{c}
0 \\
\tau\left(a^{i}, b^{i}\right)+\tau\left(b^{i}, c^{i}\right)-\tau\left(a^{i}, c^{i}\right)
\end{array}\right] \geq 0 \quad \text { for } i=1, \ldots, L_{3} .
$$

Inequalities (a) and (b) follow from (1a) and (1b), respectively. The triangle inequality is reflected in (c). Nonnegativity of $\Omega$ follows from (b), (c), and irreflexivity of $\succ$. Symmetry of $\Omega$ is already reflected in definition of $\tau$.

We are to establish that the system of linear inequalities (a), (b), and (c) has a $\rho$ solution. Therefore, a poset $(X, \succ)$ has a semimetric threshold representation. Suppose on the contrary that there is no $\rho$ solution. Then it follows from Lemma 1 that there are nonnegative integers $\alpha_{1}$ for $i=$ $1, \ldots, L_{1}, \beta_{i 1}$ for $i=1, \ldots, L_{2}, \beta_{i 2}$ for $i=1, \ldots, L_{2}$, and $\gamma_{i}$ for $i=1, \ldots, L_{3}$ such that $\alpha_{j}>0$ for some $1 \leq j \leq L_{1}$, and the following LD equation holds:

$$
\begin{gathered}
\sum_{i=1}^{L_{1}} \alpha_{i}\left[\begin{array}{c}
\theta\left(x^{i}\right)-\theta\left(y^{i}\right) \\
-\tau\left(x^{i}, y^{i}\right)
\end{array}\right]+\sum_{i=1}^{L_{2}} \beta_{i 1}\left[\begin{array}{c}
\theta\left(z^{i}\right)-\theta\left(w^{i}\right) \\
\tau\left(z^{i}, w^{i}\right)
\end{array}\right]+\sum_{i=1}^{L_{2}} \beta_{i 2}\left[\begin{array}{c}
\theta\left(w^{i}\right)-\theta\left(z^{i}\right) \\
\tau\left(z^{i}, w^{i}\right)
\end{array}\right] \\
+\sum_{i=1}^{L_{3}} \gamma_{i}\left[\begin{array}{c}
0 \\
\tau\left(a^{i}, b^{i}\right)+\tau\left(b^{i}, c^{i}\right)-\tau\left(a^{i}, c^{i}\right)
\end{array}\right]=0 .
\end{gathered}
$$


Let $m=\sum \alpha_{i}, \ell=\sum \beta_{i 1}+\sum \beta_{i 2}$, and $k=\sum \gamma_{i}$. Then $m>0, m=\ell+k$, and $0 \leq \ell \leq k$, because $\tau$ s are unit vectors and, for all $x, y, z, w \in X$, $\tau(x, y) \neq \tau(z, w)$ if $x \succ y$ and $z \sim w$.

List the elements of $\succ, \sim$, and $X \times X \times X$ with $\alpha_{i}$ repeats for $\left(x^{i}, y^{i}\right)$, $\beta_{i 1}$ repeats for $\left(z^{i}, w^{i}\right), \beta_{i 2}$ repeats for $\left(w^{i}, z^{i}\right)$, and $\gamma_{i}$ repeats for $\left(a^{i}, b^{i}, c^{i}\right)$, and enumerate them as

$$
\begin{array}{ll}
\left(x_{1}^{*}, y_{1}^{*}\right), \ldots,\left(x_{m}^{*}, y_{m}^{*}\right) & \text { for } \succ \\
\left(z_{1}^{*}, w_{1}^{*}\right), \ldots,\left(z_{\ell}^{*}, w_{\ell}^{*}\right) & \text { for } \sim, \\
\left(a_{1}^{*}, b_{1}^{*}, c_{1}^{*}\right), \ldots,\left(a_{k}^{*}, b_{k}^{*}, c_{k}^{*}\right) & \text { for } X \times X \times X .
\end{array}
$$

Then the LD equation is described as follows:

$$
\begin{aligned}
& \sum_{i=1}^{m}\left[\begin{array}{c}
\theta\left(x_{i}^{*}\right)-\theta\left(y_{i}^{*}\right) \\
-\tau\left(x_{i}^{*}, y_{i}^{*}\right)
\end{array}\right]+\sum_{i=1}^{\ell}\left[\begin{array}{c}
\theta\left(z_{i}^{*}\right)-\theta\left(w_{i}^{*}\right) \\
\tau\left(z_{i}^{*}, w_{i}^{*}\right)
\end{array}\right] \\
& +\sum_{i=1}^{k}\left[\begin{array}{c}
0 \\
\tau\left(a_{i}^{*}, b_{i}^{*}\right)+\tau\left(b_{i}^{*}, c_{i}^{*}\right)-\tau\left(a_{i}^{*}, c_{i}^{*}\right)
\end{array}\right]=0 .
\end{aligned}
$$

In what follows, we show that the LD equation contradicts transitivity of $\succ$. We have two cases to examine: $\ell=0 ; 0<\ell \leq k$.

Case $1 \quad(\ell=0) \quad$ The first $n$ rows of the LD equation is

$$
\sum_{i=1}^{m} \theta\left(x_{i}^{*}\right)=\sum_{i=1}^{m} \theta\left(y_{i}^{*}\right)
$$

which gives that the sequence $x_{1}^{*}, \ldots, x_{m}^{*}$ is a permutation of the sequence $y_{1}^{*}, \ldots, y_{m}^{*}$. Since $x_{i}^{*} \succ y_{i}^{*}$ for $i=1, \ldots, m$, it is easily seen that transitivity of $\succ$ is violated.

Case $2 \quad(0<\ell \leq k) \quad$ With no loss of generality, we assume that $\tau\left(z_{i}^{*}, w_{i}^{*}\right)=$ $\tau\left(a_{i}^{*}, c_{i}^{*}\right)$ for $i=1, \ldots, \ell$. Let $I_{0}=\{1, \ldots, \ell\}$. List the elements from the set $\left\{\tau\left(a_{1}^{*}, b_{1}^{*}\right), \ldots, \tau\left(a_{\ell}^{*}, b_{\ell}^{*}\right), \tau\left(b_{1}^{*}, c_{1}^{*}\right), \ldots, \tau\left(b_{\ell}^{*}, c_{\ell}^{*}\right)\right\}$ that have no identical vector in $\tau\left(x_{1}^{*}, y_{1}^{*}\right), \ldots, \tau\left(x_{m}^{*}, y_{m}^{*}\right)$, and enumerate them as $\tau^{1}, \ldots, \tau^{\ell_{1}}$. If there is an $1 \leq i^{\prime} \leq \ell$ such that

$$
\begin{array}{ll}
\tau\left(a_{i^{\prime}}^{*}, b_{i^{\prime}}^{*}\right)=\tau\left(x_{j^{\prime}}^{*}, y_{j^{\prime}}^{*}\right) & \text { for some } 1 \leq j^{\prime} \leq m, \\
\tau\left(b_{i^{\prime}}^{*}, c_{i^{\prime}}^{*}\right)=\tau\left(x_{j^{\prime \prime}}^{*}, y_{j^{\prime \prime}}^{*}\right) & \text { for some } 1 \leq j^{\prime \prime} \leq m,
\end{array}
$$

then $a_{i^{\prime}}^{*} \succ b_{i^{\prime}}^{*}$ and $b_{i^{\prime}}^{*} \succ c_{i^{\prime}}^{*}$, so by transitivity of $\succ, a_{i^{\prime}}^{*} \succ c_{i^{\prime}}^{*}$. Since $\tau\left(a_{i^{\prime}}^{*}, c_{i^{\prime}}^{*}\right)=$ $\tau\left(z_{i^{\prime}}, w_{i^{\prime}}^{*}\right)$, we obtain a contradiction $a_{i^{\prime}}^{*} \sim c_{i^{\prime}}^{*}$. Hence $\ell \leq \ell_{1} \leq 2 \ell$.

By the LD equation, there is a sequence of $\ell_{1}$ vectors from the set $\left\{\tau\left(a_{\ell+1}^{*}, c_{\ell+1}^{*}\right), \ldots, \tau\left(a_{k}^{*}, c_{k}^{*}\right)\right\}$ that is identical to the sequence $\tau^{1}, \ldots, \tau^{\ell_{1}}$. Thus $2 \ell \leq k$. With no loss of generality, we assume that $\tau^{i}=\tau\left(a_{\ell+i}^{*}, c_{\ell+i}^{*}\right)$ for $i=1, \ldots, \ell_{1}$. Thus let $I_{1}=\left\{\ell+1, \ldots, \ell+\ell_{1}\right\}$. 
Next we construct a set $I_{2}=\left\{\ell+\ell_{1}+1, \ldots, \ell+\ell_{1}+\ell_{2}\right\}$ of indices as follows. List the elements from the set

$$
\left\{\tau\left(a_{\ell+1}^{*}, b_{\ell+1}^{*}\right), \ldots, \tau\left(a_{\ell+\ell_{1}}^{*}, b_{\ell+\ell_{1}}^{*}\right), \tau\left(b_{\ell+1}^{*}, c_{\ell+1}^{*}\right), \ldots, \tau\left(b_{\ell+\ell_{1}}^{*}, c_{\ell+\ell_{1}}^{*}\right)\right\}
$$

that have no identical vector in $\tau\left(x_{1}^{*}, y_{1}^{*}\right), \ldots, \tau\left(x_{m}^{*}, y_{m}^{*}\right)$, and enumerate them as $\tau^{\ell_{1}+1}, \ldots, \tau^{\ell_{1}+\ell_{2}}$. With no loss of generality, we assume that $\tau^{\ell_{1}+i}=$ $\tau\left(a_{\ell+\ell_{1}+i}^{*}, c_{\ell+\ell_{1}+i}^{*}\right)$ for $i=1, \ldots, \ell_{2}$. Of course, we may have $I_{2}=\emptyset$, i.e., $\ell_{2}=0$. If this is the case, we stop. Otherwise, we continue the recursive construction of $I_{3}, \ldots, I_{m^{\prime}}$ in a similar manner until $I_{m^{\prime}}$ becomes empty. Since $X$ is finite, $m^{\prime}$ is also finite.

Now we have that, for $i=1, \ldots, m^{\prime}$,

$$
I_{i}=\left\{\ell+\ell_{1}+\cdots+\ell_{i-1}+1, \ldots, \ell+\ell_{1}+\cdots+\ell_{i}\right\},
$$

where $\ell_{0}=\ell_{m^{\prime}}=0$. We observe that, for $i=1, \ldots, m^{\prime}-1$, there is a distinct $j^{\prime} \in I_{i-1}$ for every $j \in I_{i}$ such that either $\tau\left(a_{j^{\prime}}^{*}, b_{j^{\prime}}^{*}\right)=\tau\left(a_{j}^{*}, c_{j}^{*}\right)$ or $\tau\left(b_{j^{\prime}}^{*}, c_{j^{\prime}}^{*}\right)=\tau\left(a_{j}^{*}, c_{j}^{*}\right)$.

Since $I_{m^{\prime}}=\emptyset$, we obtain that, for all $i \in I_{m^{\prime}-1}$, there are $1 \leq j^{\prime} \leq m$ and $1 \leq j^{\prime \prime} \leq m$ such that

$$
\begin{aligned}
& \tau\left(a_{i}^{*}, b_{i}^{*}\right)=\tau\left(x_{j^{\prime}}^{*}, y_{j^{\prime}}^{*}\right), \\
& \tau\left(b_{i}^{*}, c_{i}^{*}\right)=\tau\left(x_{j^{\prime \prime}}^{*}, y_{j^{\prime \prime}}^{*}\right),
\end{aligned}
$$

so that $a_{i}^{*} \succ b_{i}^{*}$ and $b_{i}^{*} \succ c_{i}^{*}$. Thus, by transitivity of $\succ, a_{i}^{*} \succ c_{i}^{*}$ for all $i \in I_{m^{\prime}-1}$, so that, for all $i \in I_{m^{\prime}-2}$,

$$
\begin{array}{ll}
\text { either } & \tau\left(a_{i}^{*}, b_{i}^{*}\right)=\tau\left(a_{i^{\prime}}^{*}, c_{i^{\prime}}^{*}\right) \quad \text { for some } i^{\prime} \in I_{m^{\prime}-1}, \\
\text { or } & \tau\left(a_{i}^{*}, b_{i}^{*}\right)=\tau\left(x_{j^{\prime}}^{*}, y_{j^{\prime}}^{*}\right) \text { for some } 1 \leq j^{\prime} \leq m,
\end{array}
$$

and

$$
\begin{array}{lll}
\text { either } & \tau\left(b_{i}^{*}, c_{i}^{*}\right)=\tau\left(a_{i^{\prime \prime}}^{*}, c_{i^{\prime \prime}}^{*}\right) & \text { for some } i^{\prime \prime} \in I_{m^{\prime}-1}, \\
\text { or } & \tau\left(b_{i}^{*}, c_{i}^{*}\right)=\tau\left(x_{j^{\prime \prime}}^{*}, y_{j^{\prime \prime}}^{*}\right) & \text { for some } 1 \leq j^{\prime \prime} \leq m .
\end{array}
$$

Therefore, $a_{i}^{*} \succ c_{i}^{*}$ for all $i \in I_{m^{\prime}-2}$. This process continues up to $I_{0}$ backwardly, so that we can conclude that $a_{i}^{*} \succ c_{i}^{*}$ for all $i \in I_{0} \cup I_{1} \cup \cdots I_{m^{\prime}-1}$. However, $a_{i}^{*} \sim c_{i}^{*}$ for all $i \in I_{0}$. This is a contradiction. This completes the proof.

\section{Conclusion}

This paper proved that a finite poset has a semimetric threshold representation. However, our proof of the representation theorem is not constructive. It remains an open problem to give a constructive proof, which may also 
answer a question whether arbitrary posets have semimetric threshold representations.

\section{References}

Abbas, M. and Vincke, Ph. (1993) Preference structures and threshold models. Journal of Multi-Criteria Decision Making 2, 171-178.

Agoev, R. and Aleskerov, F. (1993) Interval choice: classic and general cases. Mathematical Social Sciences 26, 249-272.

Bridges, D.S. (1983) Numerical representation of intransitive preferences on a countable set. Journal of Economic Theory 30, 213-217.

Diaye, M-A. (1999) Variable intervals model. Mathematical Social Sciences $\mathbf{3 8}, 21-33$.

Fishburn, P.C. (1970) Utility Theory for Decision Making Wiley, New York.

Herrero, C. and Subiza, B. (1999) Set-valued utilities for strict partial orders. Journal of Mathematical Psychology 43, 433-440.

Nakamura, Y. (2000) Threshold models for comparative probability on finite sets. Journal of Mathematical Psychology 44, 353-382.

Nakamura, Y. (2001) Real interval representations. Journal of Mathematical Psychology (to appear).

Rodríguez-Palmero, C. (1997) A representation of acyclic preferences. Economics Letters 54, 143-146.

Subiza, B. (1994) Numerical representations of acyclic preferences. Journal of Mathematical Psychology 38, 467-476. 